\title{
Generalization of the Wall Theorem to Out-of-equilibrium Conditions
}

\author{
Ignacio Urrutia, ${ }^{*}$ Iván E. Paganini, and Claudio Pastorino \\ Departamento de Física de la Materia Condensada, Centro Atómico Constituyentes, \\ CNEA, Av.Gral. Paz 1499, 1650 Pcia. de Buenos Aires, Argentina and \\ Instituto de Nanociencia y Nanotecnología, CONICET-CNEA, CAC.
}

\begin{abstract}
The well-known Wall theorem states a simple and precise relation among temperature, pressure and density of a fluid at contact with a confining hard wall in thermodynamic equilibrium. In this Communication we develop an extension of the Wall theorem to out-of-equilibrium conditions, providing an exact relation between pressure, density and temperature at the wall, valid for strong non-equilibrium situations. We derive analytically this Non-equilibrium Wall theorem for stationary states and validate it with non-equilibrium event-driven molecular-dynamics simulations. We compare the analytical expression with simulations by direct evaluation of temperature, density and pressure on the wall in linear regime, medium and very strong out-of-equilibrium conditions of a nanoconfined liquid under flow in stationary state, presenting viscous heating and heat transport. The agreement between theory and simulation is excellent, allowing for a conclusive verification. In addition, we explore the degree of accuracy of using the equilibrium Wall theorem and different expressions for the local temperature, employed in non-equilibrium molecular-dynamics simulations.
\end{abstract}

\section{INTRODUCTION}

Very few exact relationships are available in the context of inhomogeneous fluids. One of such is the contact theorem also known as the Wall theorem (WT), which establishes an ideal-gas-like equation of state for the intensive properties of a fluid in thermodynamic equilibrium, at contact with a wall. The WT applies to fluids confined by hard walls that constrain the region of the space occupied by the fluid through an external zero-infinite potential. The first version of the WT[1] was derived for a fluid in contact with a planar wall or, more precisely, filling a half-space. That relation can be written as

$$
P_{w}=\rho_{w} k T,
$$

where $P_{w}$ is the pressure on the wall, $\rho_{w}$ is the density at contact with the wall, $T$ is the temperature of the system and $k$ the Boltzmann constant. In this particular planar case, the geometry of the system imposes also the extra condition $P_{w}=P$, being $P$ the pressure of the bulk fluid.[1-3]

The WT, expressed in Eq. (1), also concerns to fluids constrained by curved walls as spheres and cylinders. [46] For the application of Eq. (1) to non-planar walls, it becomes necessary to introduce the dividing surface, which fixes the position of the surface where the pressure of the fluid acts on the vessel walls. As a matter of fact, Eq. (1) is the expression of the WT for constantcurvature surfaces, written for a dividing surface which coincides with the position of the discontinuity of the hard wall-fluid potential. The choice for the location of the dividing surface can be different, but a transformation between them is straightforward.[7, 8] WT applies, for example, to fluids confined in pores both of simple[911] and complex,[12] shapes. It was used under a broad

*Electronic address: iurrutia@cnea.gov.ar variety of conditions, spaning from large systems, in the thermodynamic limit, to fluid-like very small systems comprised of very few particles.[12, 13] Key aspects of the curvature dependence of the surface free energy in confined fluids were revealed using the WT by adopting different approaches as density functional theories,[14-16] molecular dynamics[11] and virial series.[7, 17] Several formulations of the WT have been developed along time. For example, a WT was postulated for fluids composed by charged particles in contact with charged walls.[18-20]

The interest in the behavior of nanoconfined fluids is evident in the fields of Micro- and Nanofluidics and in technological applications. Efficient use of fluids for heat removal or good thermal isolation at small scales, is crucial to advance in the miniaturization of current technical developments.[21, 22] The interrelation between flow regime and adsorption of gas confined in nanoporous shale is actively studied to optimize natural gas production from shale gas reservoirs.[23-25] Highly confined inhomogeneous liquids with a relatively high surface-tovolume ratio and/or under flow, are common physical situations in those areas and the interface of the fluid with the confining wall is a key aspect to understand and tailor.[26] In this work, we are interested in hard walls that induce their temperature to the confined fluid. Naturally, the thermal hard wall cannot describe in detail the properties of a real substrate. Even though, the relevance of the thermal hard wall model to study the fluxes of energy and mass in confined fluids relies in that it is a simple prescription allowing to study the system under minimal assumptions and direct analytical calculations.

In this letter we demonstrate the Non-equilibrium Wall theorem (NEWT), applied to stationary states with thermal and velocity gradients. Under flow, viscous heating and heat transfer scenarios, we also test the NEWT using event-driven molecular-dynamics simulations (MD) of fluid flow through a narrow cylindrical channel. Our results show the validity of NEWT from near-equilibrium up to very strong non-equilibrium conditions. 


\section{THEORY}

Consider a fluid system with fixed number of particles $N$ each with mass $m$, in a confined region $\mathcal{A}$ with volume $V$. $\mathcal{A}$ is enclosed by a wall or substrate which is at a temperature $T_{\text {wall }}$. If such a fluid system is in equilibrium, its temperature is everywhere $T=T_{\text {wall }}$ and the partition function for the system reads

$$
Q=\iint f\left(\mathbf{r}^{N}, \mathbf{p}^{N}\right) d \mathbf{r}^{N} d \mathbf{p}^{N}
$$

where $f\left(\mathbf{r}^{N}, \mathbf{p}^{N}\right)=C \exp [-(\phi+\psi+K) / k T]$, with $K$ the kinetic energy, $\phi$ the external potential and $\psi$ the interaction potential between particles. This function can be factorized in different exponential terms. We focus on the external potential term $\exp [-\phi / k T]$ for the case of a hard-wall external potential $\phi$. Now, we introduce the boundary indicator function $f^{(b)} \equiv \exp [-\phi / k T]=$ $\prod_{i}^{N} \Theta\left(-\left|\mathcal{A}-\mathbf{r}_{i}\right|\right)$ with $\Theta$ the Heaviside function and $|\mathcal{A}-\mathbf{r}|$ the (shortest) distance between point $\mathbf{r}$ and $\mathcal{A}$, which is zero only if $\mathbf{r} \in \mathcal{A}$. Here, $\Theta(x)=1$ if $x \geq 0$ and $\Theta(x)=0$ if $x<0$. Naturally, we define $V=$ $\int \Theta(-|\mathcal{A}-\mathbf{r}|) d \mathbf{r}$ and $A=\int \delta(|\partial \mathcal{A}-\mathbf{r}|) d \mathbf{r}$, with $A$ the surface area of $\partial \mathcal{A}$, the boundary of the system and $\delta$ stands for the Dirac delta function. The wall not only determines the boundary of the system, but it is also a thermal wall, which sets the temperature of the bouncing particles.

For non-equilibrium (NE) stationary conditions we write

$$
Q=\iint f_{\mathrm{NE}}\left(\mathbf{r}^{N}, \mathbf{p}^{N}\right) d \mathbf{r}^{N} d \mathbf{p}^{N}
$$

where $f_{\mathrm{NE}}$ can be factorized in different terms. One of them includes the hard wall external potential and is still given by $f^{(b)}$, which fixes the boundary of the system. Now, the fluid could have different temperatures, in different regions, but the thermal wall acts as a thermal reservoir which induces locally its temperature to the fluid. Essentially, the thermal wall affects the temperature of particles once they bounce on the wall. As regards non equilibrium features, the statistical distributions are in general, of course, non-trivial. We divide the complete domain $(\mathbf{r}, \mathbf{p})$ in different subsets. For our purposes it is convenient to split the momentum space among opposite directions $\hat{\mathbf{n}}_{\text {out }}=\hat{\mathbf{n}}$ and $\hat{\mathbf{n}}_{\text {in }}=-\hat{\mathbf{n}}$, where $\hat{\mathbf{n}}$ is the normal versor to $\partial \mathcal{A}$ pointing to the outward direction of $\mathcal{A}$. $\hat{\mathbf{n}}$ depends on $\mathbf{r} \in \mathcal{A}$. Thus, we introduce the position-momentum one body density distributions

$$
\begin{aligned}
\rho_{s}(\mathbf{r}, \mathbf{p})= & Q^{-1} \iint \sum_{i}^{N} \Theta\left(\mathbf{p}_{i} \cdot \hat{\mathbf{n}}_{s}\right) \delta\left(\mathbf{p}-\mathbf{p}_{i}\right) \times \\
& \delta\left(\mathbf{r}-\mathbf{r}_{i}\right) f_{\mathrm{NE}}\left(\mathbf{r}^{N}, \mathbf{p}^{N}\right) d \mathbf{r}^{N} d \mathbf{p}^{N},
\end{aligned}
$$

and $\rho(\mathbf{r}, \mathbf{p})=\rho_{\text {in }}(\mathbf{r}, \mathbf{p})+\rho_{\text {out }}(\mathbf{r}, \mathbf{p})$. The positiondependent number density distributions are $\rho_{s}(\mathbf{r})=$ $\int \rho_{s}(\mathbf{r}, \mathbf{p}) d \mathbf{p}$ with $N_{s}=\int \rho_{s}(\mathbf{r}) d \mathbf{r}$, and subsystem in$\operatorname{dex} s=$ in, out with in and out velocities, respectively. Furthermore, the usual number density is $\rho(\mathbf{r})=$ $\sum_{s} \rho_{s}(\mathbf{r})=\rho_{\text {in }}(\mathbf{r})+\rho_{\text {out }}(\mathbf{r})$. Variables without subscript correspond to the total system. Eq. (2) is the equivalent to the statistical-mechanical definition $\rho_{s}(\mathbf{r}, \mathbf{p})=$ $\left\langle\sum_{i}^{N} \delta\left(\mathbf{r}-\mathbf{r}_{i}\right) \delta\left(\mathbf{p}-\mathbf{p}_{i}\right)\right\rangle_{s}$. We note that in NE conditions it could be the case that $\rho_{\text {in }}(\mathbf{r}) \neq \rho_{\text {out }}(\mathbf{r})$, but at equilibrium, the relation $\rho_{\text {in }}(\mathbf{r})=\rho_{\text {out }}(\mathbf{r})=\rho(\mathbf{r}) / 2$ must hold.

Number densities and other mean magnitudes of the fluid on the wall, labeled as $w$, can be written as:

$$
\begin{aligned}
\rho_{w, s} & =A^{-1} \iint \rho_{s}(\mathbf{r}, \mathbf{p}) \delta(|\partial \mathcal{A}-\mathbf{r}|) d \mathbf{r} d \mathbf{p} \\
{[X]_{s} \rho_{w, s} } & =A^{-1} \iint X \rho_{s}(\mathbf{r}, \mathbf{p}) \delta(|\partial \mathcal{A}-\mathbf{r}|) d \mathbf{r} d \mathbf{p},
\end{aligned}
$$

where $X$ is a function of one particle position and momentum $(\mathbf{r}, \mathbf{p})$. For example, the mean velocity in a tangential direction $\hat{\mathbf{t}}$ of particles coming out of the wall is $[\mathbf{p} \cdot \hat{\mathbf{t}}]_{\text {out }} / m$. The total mean value on the wall is recovered by the expression $[X]=$ $\left([X]_{\text {in }} \rho_{w, \text { in }}+[X]_{\text {out }} \rho_{w, \text { out }}\right) / \rho_{w}$.

We point out that in non-equilibrium conditions different forms of measuring the temperature may give different results.[27, 28] A frequently used prescription for calculating the temperature in MD simulations is through the square of the particles velocity, relative to the stream velocity. It is referred as the kinetic temperature. $[29,30]$ On the other hand, we can evaluate the temperature using the kinetic energy in different characteristic directions that do not present a net flux of particles.[31] Here, we follow this approach to introduce the temperature measured in the normal direction

$$
k T_{w, s}^{(n)} m=\left[(\mathbf{p} \cdot \hat{\mathbf{n}})^{2}\right]_{s},
$$

associated with the velocity normal to the wall. We may note that, as it could be the case that $\rho_{w \text {,in }} \neq \rho_{w \text {,out }}$, then it could also happen that the temperature of the fluid on the wall, $T_{w, \text { in }}^{(n)}$ and $T_{w \text {,out }}^{(n)}$, were different to $T_{\text {wall }}$. Alongside, if equilibrium is established there is a unique temperature for the entire system $T=T_{\text {wall }}=T_{w \text {,in }}^{(n)}=$ $T_{w, \text { out }}^{(n)}$.

The pressure on the wall is the force exerted by the fluid per unit area and normal to the wall. It results from the mean value of momentum transfer (flux) between the fluid and the wall substrate in the normal direction. Taking into account the number of incident (in) and scattered (out) particles with normal velocity $\mathbf{v} \cdot \hat{\mathbf{n}}$ and the momentum they transfer to the wall, we obtain

$$
P_{w, s}=A^{-1} \iint \frac{(\mathbf{p} \cdot \hat{\mathbf{n}})^{2}}{m} \rho_{s}(\mathbf{r}, \mathbf{p}) \delta(|\partial \mathcal{A}-\mathbf{r}|) d \mathbf{r} d \mathbf{p} .
$$

Expression (6) requires a derivation that will be given in the next paragraph. A comparison between Eqs. (4, 
5) and (6) shows that normal kinetic temperature $T_{w, s}^{(n)}$ plays a special role. Here we collect results from Eq. (3) to $(6)$, to obtain that

$$
P_{w, s}=\rho_{w, s} k T_{w, s}^{(n)} .
$$

It addition, it can be verified that the total momentum flux corresponds to the sum over $s$-index, taking the values in and out. Then, the pressure on the wall is $P_{w}=P_{w, \text { out }}+P_{w, \text { in }}$. In this way we obtain the main result of the present work:

$$
P_{w}=\rho_{w} k T_{w}^{(n)} .
$$

Eq. (8) expresses the generalization to non-equilibrium conditions of the Wall theorem for the pressure. It simply states that under NE, even when the different forms of measuring the kinetic temperature are non-equivalent, the WT still applies, if the temperature $T$ is replaced by the kinetic temperature measured in the direction normal to the wall.

Derivation: Instead of focusing on the momentum flux given in Eq. (6), we analyze now a more general case: the flux of a generic quantity $Y$. We consider the skin $\partial \mathcal{A}^{\epsilon}$ of $\mathcal{A}$, that extends a small depth $\epsilon$ from $\partial \mathcal{A}$, towards the inner direction, and a small interval of momentum in the normal direction $\left(p_{z}, p_{z}+\Delta p_{z}\right)$. The normal outward versor for any point in $\partial \mathcal{A}^{\epsilon}$ is $\hat{z} \equiv \hat{\mathbf{n}}_{\text {out }}$. Each particle in $\partial \mathcal{A}^{\epsilon}$ has a constant velocity to a good approximation. This approximation becomes better for a progressively smaller $\epsilon$. The number of particles in $\partial \mathcal{A}^{\epsilon}$ that will collide with the wall per unit time, having normal momentum between $p_{z}$ and $p_{z}+\Delta p_{z}$ along an small time interval $\tau$ is given by

$$
\frac{1}{\tau} \iiint_{-\tau p_{z} / m}^{0} \rho_{\text {out }}(\mathbf{r}, \mathbf{p}) J_{\mathbf{r}} J_{\mathbf{p}} d z \Delta p_{z} d \mathbf{r}_{\bar{z}} d \mathbf{p}_{\bar{z}} .
$$

We write $d \mathbf{r}=J_{\mathbf{r}} d z d \mathbf{r}_{\bar{z}}$ to explicitly separate the differential in $z$ direction from the other spatial directions packed in $d \mathbf{r}_{\bar{z}}$, with $J$ the jacobian, and assume $\epsilon>\tau p_{z} / m$. The value $z=0$ is the wall position. The mean flux of $Y(\mathbf{r}, \mathbf{p})$ is then

$$
\frac{1}{\tau} \iiint_{-\tau p_{z} / m}^{0} Y \rho_{\text {out }}(\mathbf{r}, \mathbf{p}) J_{\mathbf{r}} J_{\mathbf{p}} d z \Delta p_{z} d \mathbf{r}_{\bar{z}} d \mathbf{p}_{\bar{z}} .
$$

Specifically, this is the flux of $Y$ towards the wall exerted by particles with normal momentum in the range $\left(p_{z}, p_{z}+\Delta p_{z}\right)$. Now we integrate in $d z$, after that we take the limits $\lim _{\epsilon \rightarrow 0} \lim _{\tau \rightarrow 0}$ and finally we replace $\Delta p_{z}$ by $d p_{z}$ to integrate in $d p_{z}$, which reduces Eq. (9) to

$$
\iiint \frac{\mathbf{p} \cdot \hat{\mathbf{n}}}{m} Y_{w}(\mathbf{p}) \rho_{w, \text { out }}(\mathbf{p})\left(J_{\mathbf{r}} J_{\mathbf{p}}\right)_{w} d p_{n} d \mathbf{r}_{\bar{n}} d \mathbf{p}_{\bar{n}} .
$$

We cast this to a volumetric integral in space, following Eq. (4), and collect the result for both directions, $s=$ in and $s=$ out, to obtain

$$
j_{w, s}(Y)=\frac{1}{A} \iint \frac{\mathbf{p} \cdot \hat{\mathbf{n}}}{m} Y \rho_{s}(\mathbf{r}, \mathbf{p}) \delta(|\partial \mathcal{A}-\mathbf{r}|) d \mathbf{r} d \mathbf{p} .
$$

$j_{w, s}(Y)$ is the flux of $Y$ per unit time and area between the fluid and the wall for the $s$-subsystem, in the direction normal to the wall. The deduction for $s=$ in is presented in the Supplementary Material (SM). Collecting results from Eqs. $(3,4)$ we obtain the general relation

$$
j_{w, s}(Y)=[\mathbf{p} \cdot \hat{\mathbf{n}} Y / m]_{s} \rho_{w, s} .
$$

Pressure due to outcoming (incoming) particles is the normal momentum flux per unit time and area. Thus, choosing $Y=\mathbf{p} \cdot \hat{\mathbf{n}}$ to replace in Eq. (12), we obtain the pressure $P_{w, s}=j_{w, s}(\mathbf{p} \cdot \hat{\mathbf{n}})=\rho_{w, s} k T_{w, s}^{(n)}$, i.e. the expression in Eq. (7). This completes our derivation of the Wall theorem for the pressure in out-of-equilibrium, stationary conditions.

Before proceeding to the numerical test of the NEWT by MD simulations, we would like to point out a further generalization based on the given derivation. On one hand, Eqs. (3, 4, 6) and (8) correspond to mean values over the boundary $\partial \mathcal{A}$. However, the identity should be valid not only for the integrals but also for the integrands in Eqs. (4) and (11), once we replace $X \rightarrow \mathbf{p} \cdot \hat{\mathbf{n}} Y / m$. Therefore, the derivations of Eqs. $(3,4,6)$ and (8) can be also performed for a small patch in $\partial \mathcal{A}$ around a point $\mathbf{r}$ of the surface, instead of using the complete boundary. This is the case, at least, for any smooth region of $\partial \mathcal{A}$, where the direction normal to the surface is well-defined. Under this condition, following a similar approach to that used above, and choosing $Y=\mathbf{p} \cdot \hat{\mathbf{n}}$, a local version of Eqs. $(3,4,6)$ and $(8)$ is found. This local NEWT for the pressure is given by

$$
P_{w}(\mathbf{r})=\rho_{w}(\mathbf{r}) k T_{w}^{(n)}(\mathbf{r}) .
$$

This very insteresting expression should be the subject of additional work and it is beyond the scope of this paper.

\section{SIMULATION TEST}

Out-of-equilibrium event-driven molecular dynamics simulations were performed to cross-check the validity of the NEWT in Eq. (8). We study a fluid flowing through a narrow cylindrical channel with a thermostated wall. We chose event-driven molecular dynamics simulations because they provide a precise definition and straight implementation of hard walls, which allow for a clean comparison with the analytic results.[32] The chosen mechanism to fix the wall temperature is the well-known thermalwall thermostat, described in detail elsewhere.[33, 34] The particles of the fluid interact through a square well potential with parameters $\sigma=1, \varepsilon=1$ and $\lambda=0.5 \sigma$. In the axial direction, an external constant body force $F$, is applied on each particle to induce a liquid flow. We provide more details of the simulations in SM. We analyze the system behavior as a function of the external force, by increasing progressivelly the flow rate and therefore, the local viscous heating in the fluid. We point out that the particles are thermostated only at the wall, allowing 


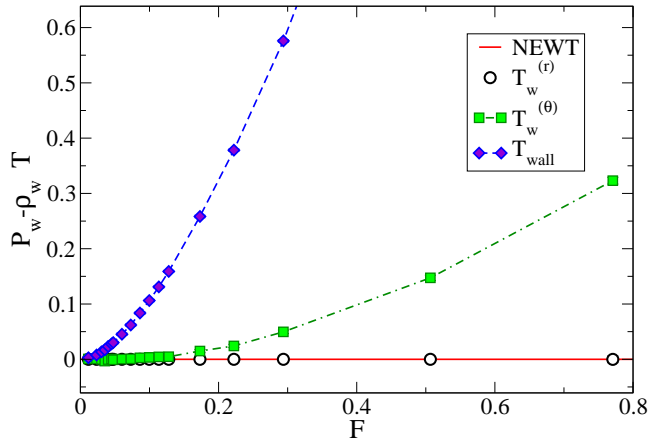

Figure 1: Comparison of the analytical expression of the NEWT (Eq. 8) and MD results, as a function of external driving $F$. Higher values of $F$ mean more extreme nonequilibrium conditions. The red line indicates the analytical result of Eq. (8) in the form $P_{w}-\rho_{w} T_{w}^{(r)} \equiv 0$. Simulation results, in open circles, show an excellent agreement for the complete range of external driving. Blue diamonds correspond to the use of the wall temperature, i.e. the equilibrium version of the Wall theorem and the curve with green squares is obtained by using the temperaure calculated in the angular direction.

for local heating of the bulk fluid when it is forced to flow. The mean number density of the liquid is set to $0.6 \sigma^{-3}$, and the wall temperature to $T_{\text {wall }}=1.3 \varepsilon / k$ for all the cases. As we use reduced units for the presentation of simulation results, they will be omitted in the following paraghraps and figures (see SM for more details), where we also fix the Boltzmann constant to $k=1$. The fluid temperature was measured by averaging the kinetic energy of the particles. For simplicity, this temperature was calculated with the kinetic energy in $\theta$ direction, in which the fluid has no streaming velocity. The pressure on the wall is given by the flux of momentum normal to the wall, per unit time and surface area. The details of this calculation are given in Sec. II. C. in the SM.

We present the comparison of the analytical NEWT expression (Eq. 8) with simulation results in Fig. 1. We calculated for the system in steady state, the mean values of pressure, density and kinetic temperatures of the particles on the wall for different external forces $F$. In Fig. 1 we plot the pressure on the wall $P_{w}$ minus number density on the wall $\rho_{w}$ times characteristic temperature, i.e. an alternative expression for the NEWT in Eq. 8 . There, the temperature was measured using three different prescriptions: the temperature of the wall as fixed in the simulations, and the temperature on the wall following two different directions of the kinetic energy in the cylindrical nanochannel, normal to the surface $\hat{\mathbf{r}}$ and angular $\hat{\boldsymbol{\theta}}$. The zero abcisa red line corresponds to the analytical prediction of the NEWT from Eq. (8) with normal temperature $T_{w}^{(r)}$. In this way, the proximity to zero value indicates the level of agreement between theory and simulation. The values of $T_{w}^{(r)}$, as calculated from the simulations (open circles), verify the validity of the NEWT along the entire studied range of driving forces. The simulations provide an excelent agreement with the expression obtained for the NEWT in Eq. (8). If we use the wall temperature $T_{\text {wall }}$ instead, it deviates from the exact behavior even for small forces. Temperature $T_{w}^{(\theta)}$ is used as a measure of the total temperature of the fluid at the wall. Results show that, as the force is increased beyond $F \approx 0.2$, the difference between $P_{w}$ and $\rho_{w} T_{w}^{(\theta)}$ becomes evident, indicating the end of the near local-equilibrium regime for the studied system.

In SM we show MD results for the behavior of $P_{w}$ vs. $\rho_{w}$ at constant wall temperature for steady-state flow. There, the NEWT is verified and the quality of different approximations for the temperature at the wall are tested. In Fig. 2 the demeanor of the confined fluid for different external forces $F$ is shown to illustrate the general behavior of the fluid under increasingly higher driving. From left to right panels in Fig. 2, velocity, density and temperature profiles are shown. As we expect, for larger forces, higher fluxes and more pronounced velocity gradients are observed (Panel A). At higher fluxes a significant change in the density profile near the wall is observed (see Panel B, in Fig. 2), attributed to larger differences in temperatures between the central region of the channel and the wall, due a higher viscous heating (Panel C). This is a physical situation observed for the limit of very high flow rate in the simulations, but expectable in nanofluidics or thermal microdevices such as heat exchangers or dissipators. The difference in the temperature between fluid and wall could arise from viscous heating of the flowing liquid, as in our simulations, or because the liquid is in contact with sources at two different temperatures. Independently of the precise physical origin of the difference in temperatures the NEWT is valid and relevant to shed light on the behavior of thermodynamic quantities at the fluid-wall interface.

We point out that even when we tested the NEWT with flow of a SW model fluid confined in a cylindrical channel, the derivation of the NEWT is general. It applies to any fluid confined by hard walls of any geometry and under non-equilibrium stationary states. The chosen system allows for the accurate testing of the NEWT in relevant and complex set of conditions, such as a curved confining wall, liquid flow and temperature gradients arising from viscous heating. The NEWT opens the possibility of analysing accurately the difference between equilibrium and stationaty out-of-equilibrium states of highly confined inhomogeneous systems.

\section{FINAL REMARKS}

The Wall theorem, applied up to now to fluids in equilibrium, was extended to out-of-equilibrium conditions, and including nanoconfined systems. This extension is demonstrated theoretically and validated numerically using event-driven molecular dynamics under strong nonequilibrium situations. We test our analytic results for a fluid flowing through a small cylindrical channel with 

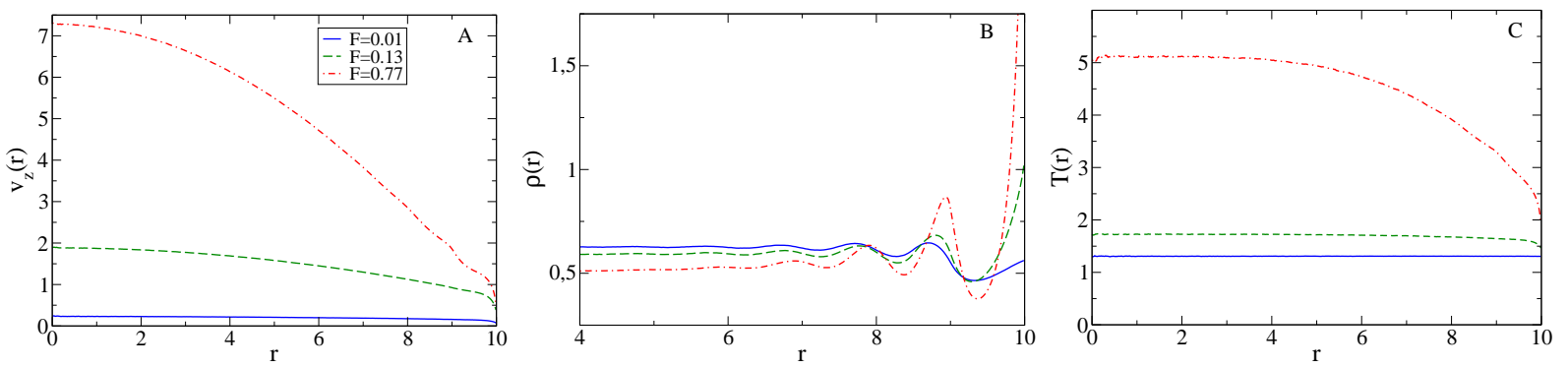

Figure 2: Profiles for $v(r), \rho(r)$ and $T(r)$ in the radial direction of the cylindrical nanochannel. Three volume force values are considered, spanning the condition close-to-equilibrium (blue curves), moderate flow (green curves) and strong out-ofequilibrium conditions (red curves). The liquid properties change significantly for the three cases. The velocity profile (Panel A) is close to a parabolic Poiseuille flow, but the slip velocity on the wall increases upon increasing flow and, at very high driving (red curves), a structure appears, following the strong layering in the density. The liquid density (Panel B) increases strongly close to the wall when driving is increased and the viscous heating is more pronounced (see Panel C).

very-high temperature and velocity gradients. We expect that this result will be relevant for theory and experiments on fluids at the nanoscale. The NEWT should be of particular interest in nanofluidics, in which many measurements of the system are done at the surface of the confining media. We emphasize, however that the result remains valid for any stationary non-equilibrium state such as microflows and even macroscopic systems, which may involve compressible non-newtonian fluids and could present temperature gradients, velocity gradients and fluid flow.

\section{Supplementary Material}

See supplementary material for the derivation of Eq. (10) for the $s=$ in case. There, we also include information about simulation details and an analysis of the evolution of $P_{w}$ vs. $\rho_{w}$ at constant wall temperature.
[1] J. L. Lebowitz, Physics of Fluids 3, 64 (1960).

[2] J. R. Henderson and F. van Swol, Molecular Physics 51, 991 (1984).

[3] D. Henderson and M. Plischke, Proceedings of the Royal Society of London. A. Mathematical and Physical Sciences 410, 409 (1987).

[4] J. R. Henderson, Molecular Physics 50, 741 (1983).

[5] J. R. Henderson, in Fluid interfacial phenomena, edited by C. A. Croxton (Wiley, New York, 1986), pp. 555-605.

[6] J. R. Henderson and J. S. Rowlinson, The Journal of Physical Chemistry 88, 6484 (1984).

[7] I. Urrutia, Phys. Rev. E 89, 032122 (2014).

[8] A. Reindl, M. Bier, and S. Dietrich, Phys. Rev. E 91, 022406 (2015).

[9] J. F. Lutsko, J. Laidet, and P. Grosfils, Journal of Physics: Condensed Matter 22, 035101 (2010).

[10] E.-Y. Kim, S.-C. Kim, and B.-S. Seong, Fluid Phase Equilibria 327, 30 (2012).

[11] I. E. Paganini, C. Pastorino, and I. Urrutia, The Journal of Chemical Physics 142, 244707 (2015).

[12] I. Urrutia, The Journal of Chemical Physics 133, 104503 (pages 26) (2010).

[13] I. Urrutia, The Journal of Chemical Physics 135, 024511 (2011), erratum: ibid. 135(9), 099903 (2011).

[14] E. M. Blokhuis and J. Kuipers, The Journal of Chemical Physics 126, 054702 (pages 10) (2007)

[15] E. M. Blokhuis, Phys. Rev. E 87, 022401 (2013).

[16] H. Hansen-Goos, The Journal of Chemical Physics 141, 171101 (2014).
[17] I. Urrutia, Phys. Rev. E 94, 022149 (2016).

[18] D. Henderson, L. Blum, and J. L. Lebowitz, Journal of Electroanalytical Chemistry and Interfacial Electrochemistry 102, 315 (1979)

[19] L. B. Bhuiyan and D. Henderson, The Journal of Chemical Physics 128, 117101 (pages 2) (2008)

[20] D. Henderson and D. Boda, Phys. Chem. Chem. Phys. 11, 3822 (2009).

[21] R. B. Schoch, J. Han, and P. Renaud, Rev. Mod. Phys. 80, 839 (2008).

[22] T. M. Squires and S. R. Quake, Rev. Mod. Phys. 77, 977 (2005).

[23] M. Kazemi and A. Takbiri-Borujeni, Energy \& Fuels 30, 8156 (2016).

[24] K. Wu, Z. Chen, X. Li, J. Xu, J. Li, K. Wang, H. Wang, S. Wang, and X. Dong, Fuel 205, 173 (2017).

[25] Y. Shen, Y. Pang, Z. Shen, Y. Tian, and H. Ge, Scientific Reports 8, 2601 (2018).

[26] H. Hu, C. Xu, Y. Zhao, K. J. Ziegler, and J. N. Chung, Scientific Reports 7, 6117 (2017).

[27] A. V. Popov and R. Hernandez, The Journal of Chemical Physics 126, 244506 (2007).

[28] P. K. Patra and R. C. Batra, Phys. Rev. E 95, 013302 (2017).

[29] X.-J. Liu, H. Hu, and P. D. Drummond, Phys. Rev. B 82, 054524 (2010).

[30] D. Frenkel and B. Smit, Understanding Molecular Simulation: From Algorithms to Applications (Academic Press, 2002). 
[31] B. Todd and P. Daivis, Nonequilibrium Molecular Dynamics: Theory, Algorithms and Applications (Cambridge University Press, 2017).

[32] M. Klement and M. Engel, The Journal of Chemical Physics 150, 174108 (2019).
[33] R. Tehver, F. Toigo, J. Koplik, and J. R. Banavar, Phys. Rev. E 57, R17 (1998).

[34] S. Taniguchi, A. Iwasaki, and M. Sugiyama, Journal of the Physical Society of Japan $\mathbf{7 7}, 124004$ (2008). 


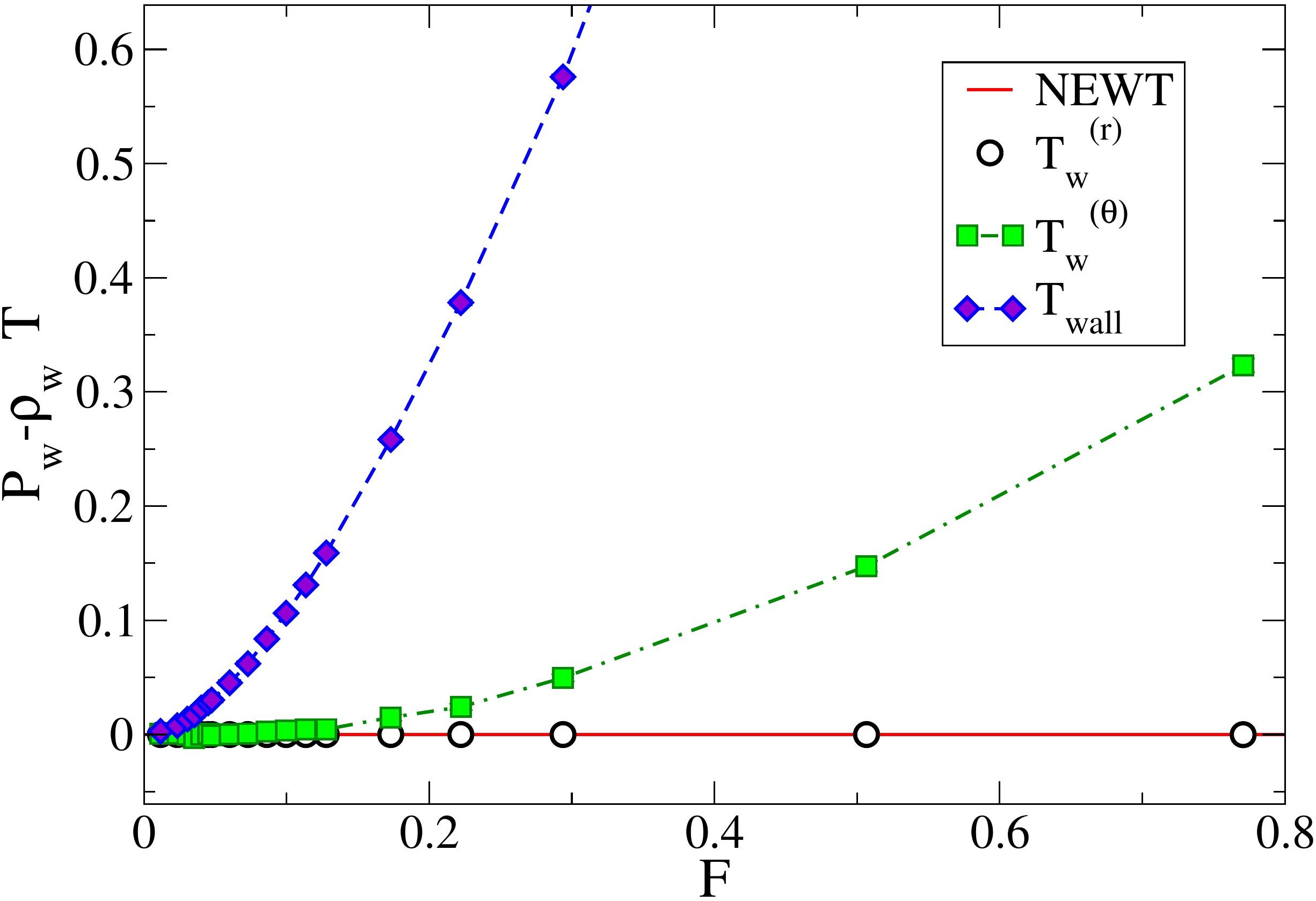




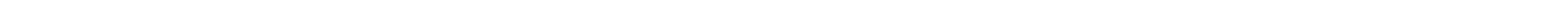



